\title{
Robotic lobectomy costs and quality of life
}

\author{
Jennifer M. Nishimura, Matthew Goodwin, Peter Kneuertz, Susan Moffatt-Bruce, Robert E. Merritt, \\ Desmond M. D'Souza
}

Division of Thoracic Surgery, Department of Surgery, The Ohio State University Wexner Medical Center, Columbus, OH 43210, USA.

Correspondence to: Dr. Desmond M. D'Souza, Division of Thoracic Surgery, The Ohio State University Wexner Medical Center, N835 Doan Hall, 410 West 10th Avenue, Columbus, OH 43210, USA. E-mail: desmond.d'souza@osumc.edu

\begin{abstract}
How to cite this article: Nishimura JM, Goodwin M, Kneuertz P, Moffatt-Bruce S, Merritt RE, D'Souza DM. Robotic lobectomy costs and quality of life. Mini-invasive Surg 2020;4:11. http://dx.doi.org/10.20517/2574-1225.2019.48
\end{abstract}

Received: 31 Oct 2019 First Decision: 2 Jan 2020 Revised: 13 Jan 2020 Accepted: 3 Feb 2020 Published: 14 Feb 2020

Science Editor: Valérie Lacroix Copy Editor: Jing-Wen Zhang Production Editor: Jing Yu

\begin{abstract}
The surgical approach for lobectomy has changed over time with recent data demonstrating that the majority are performed using a minimally invasive approach. While the use of the robotic platform for pulmonary resection has been shown to have acceptable clinical outcomes, cost and quality of life need to be considered when starting a robotic lobectomy program. In this review, we evaluate the literature on cost of robotic lobectomy and quality of life. The results suggest that early experience in a robotic lobectomy program may be associated with relatively higher index hospital costs when compared to video-assisted thoracoscopic surgery; however, with increased experience and volume, the difference may no longer be of significance. When compared with thoracotomy, the cost is comparable if not less costly and may even be profitable for the hospital. Quality of life appears to be acceptable in the early experience of robotic lobectomy.
\end{abstract}

Keywords: Robotic, thoracic surgery, lobectomy, cost, quality of life, patient reported outcomes

\section{INTRODUCTION}

The surgical approach for pulmonary lobectomy has significantly changed over time. Two decades ago, the majority of lobectomies were performed via thoracotomy. Over time, surgeons began to adopt videoassisted thoracoscopic surgery (VATS) and an increased proportion of lobectomies were performed using this minimally invasive approach. The da Vinci Surgical System (Intuitive Surgical; Sunnyvale, California, USA) later provided an alternative platform. The proportion of lobectomies after introduction 
of this system performed by thoracotomy continued to decline. One study showed that, in $2008,76.2 \%$ of lobectomies were performed using the open approach, compared with $23.4 \%$ and $<1.0 \%$ for VATS and robotic approaches, respectively ${ }^{[1]}$. In 2014, the majority of lobectomies was no longer performed via the open approach, and VATS and robotic approaches comprised $31.6 \%$ and $25.0 \%$ of lobectomies, respectively ${ }^{[1]}$. Another study demonstrated that, from 2011 to 2015, lobectomies performed by thoracotomy had an absolute decline of $11.5 \%{ }^{[2]}$. Lobectomies performed using the robotic approach had an absolute increase of $10 \%$, yet VATS only had an absolute increase of $1.5 \%{ }^{[2]}$.

While the use of the robotic platform for lobectomy is growing and its safety has been evaluated and found to be acceptable $e^{[2-5]}$, additional considerations for utilizing the robotic approach over other techniques and starting a robotic lobectomy program are still under evaluation, including costs and patient reported outcomes (PRO). A systematic review of the literature on the cost of robotic-assisted lobectomy that was performed by Singer et al.$^{[6]}$ from our institution, which included six observational studies published before 1 December 2017, found that, in general, the costs of robotic lobectomy exceed those of VATS. The studies that they reviewed were primarily based on early experiences, with the study period ranging from 2007 to 2013, and were only from the USA.

In this article, an updated review of the literature of the cost of robotic lobectomy is presented and the quality of life in these patients is reviewed.

\section{METHODS}

\section{Literature search}

An electronic literature search on PubMed was performed to identify studies that included either robotic lobectomy costs or quality of life on 9 September 2019. Search terms used included: ("cost" or "charges" or "quality of life" or "patient reported outcomes") AND ("robotic" or "robot") AND ("lobectomy" or "anatomic resection"). Abstracts from the search result were screened for relevance to include studies that evaluated costs and/or quality of life in patients undergoing robotic lobectomy. Original articles written in English were selected. Case reports and abstract-only publications were excluded. The full-text of the remaining studies were reviewed for eligibility. Additional studies were identified from reviewing the references of the studies found in the electronic literature search.

\section{RESULTS}

The literature search for costs associated with robotic lobectomy and review of its references resulted in 16 relevant articles [Table 1] from five different countries (Canada, 1; China, 2; France, 1; Italy, 1; and USA, 11$)^{[1,7-21]}$. These articles were published from 2008 to 2019 with the study period ranging from 2008 to 2017. The number of patients undergoing robotic lobectomy ranged from 12 to 2498 . All studies were observational. The majority of studies were retrospective analyses of prospectively collected data from a single institution. Other studies included one prospective observational study ${ }^{[7]}$ and four population-based cohort studies ${ }^{[1,8-10]}$. In addition to analyzing costs of patients undergoing robotic lobectomy, seven of these studies also included patients who underwent robotic segmentectomy or wedge resection ${ }^{[7,9,11,13-15,18]}$. The majority of studies reported using the da Vinci Si system. Only two studies noted the use of the $\mathrm{Xi}^{[16,17]}$. Both four-arm ${ }^{[11,13,14,17,18,20]}$ and three-arm ${ }^{[7,12,15,19,21]}$ techniques were reported. There were three relevant articles identified that studied quality of life in patients undergoing robotic lobectomy ${ }^{[21-23]}$.

\section{ROBOTIC LOBECTOMY COST}

\section{Cost definition and analysis}

Costs reported in these studies were based on the index hospitalization. There was significant heterogeneity in the definition of cost, how it was analyzed, and the detail provided of these costs. Studies reported total 


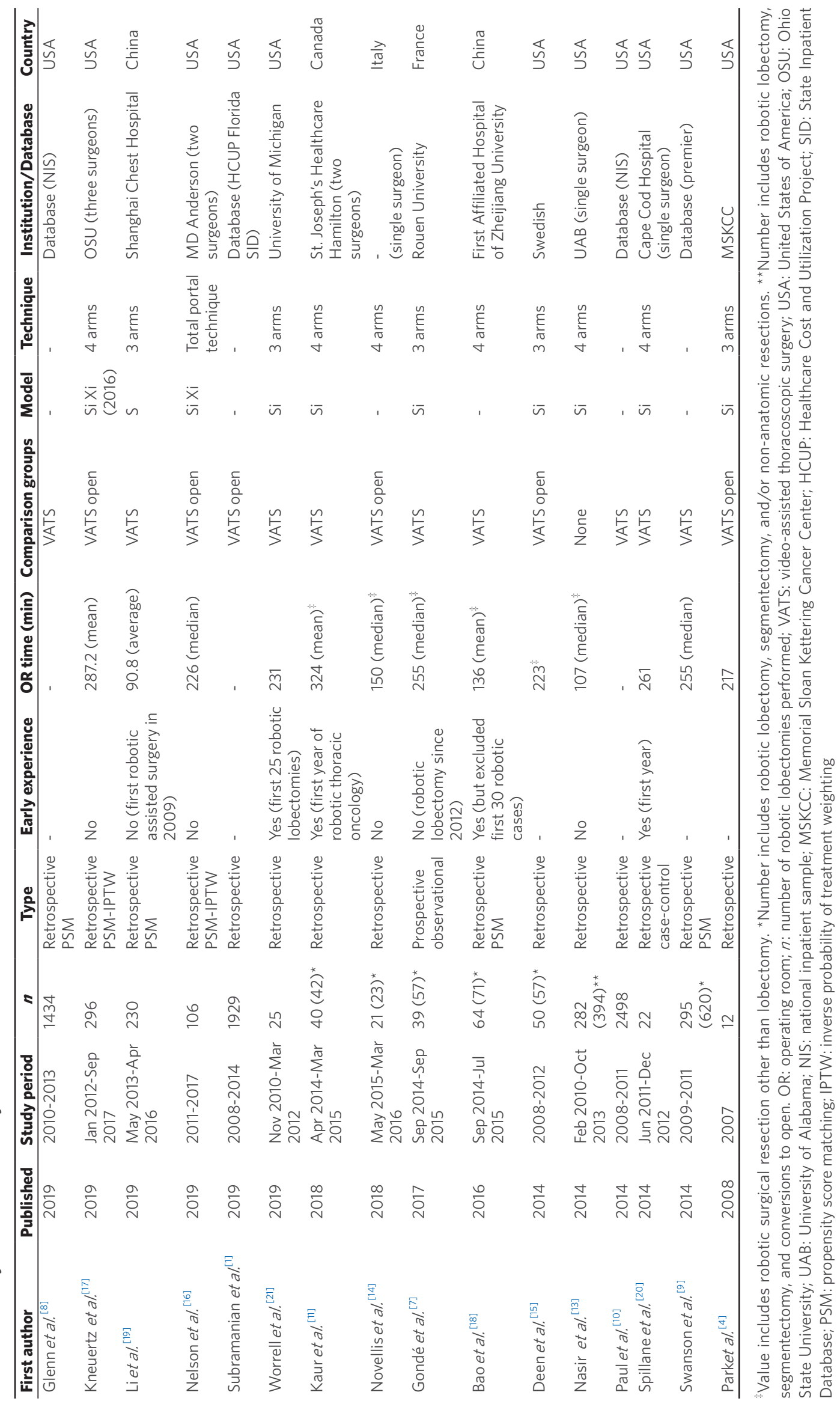


costs, direct costs, and/or indirect costs. Details on operating room (OR) charges and costs were provided by some studies. Professional fees were included in some studies, but not all. The micro-costing method was used to assess costs in the studies by Kaur et al. ${ }^{[11]}$ and Gondé et al. ${ }^{[7]}$ Relative cost, rather than absolute cost, was reported in the study by Park ${ }^{[12]}$.

Total costs were reported as the sum of indirect and direct costs in the study by Nasir et al ${ }^{[13]}$. Direct cost was defined as the cost of any items used and services provided in the care of the patient during the hospitalization. This included all operating room disposable equipment and supplies; staplers; laboratory tests; imaging studies; pharmacy items and medications; and salaries and benefits of personnel who delivered care to the patient. Indirect cost was defined as overhead cost and amortization of capital equipment and supplies and maintenance.

Robotic specific costs were defined and reported by many studies and included direct costs such as disposable instruments, drapes, and other supplies. Other robotic specific costs provided included amortized cost/capital depreciation and maintenance costs. Robot depreciation in the study by Novellis et al. ${ }^{[14]}$ was estimated from capital cost of 2 million euros plus annual maintenance of 200,000 euros divided by the number of procedures per year (400 cases) over eight years. Deen et al. ${ }^{[15]}$ calculated capital depreciation and service cost of 1200 USA dollars (USD) per case by considering four robots priced at two million USD each, performing 2403 procedures in a 22-month period. Gondé et al. ${ }^{[7]}$ calculated capital depreciation by dividing the sum of the purchase price and maintenance cost by the number of surgical procedures per year multiplied by the depreciation period. In the study by Nelson et al. ${ }^{[16]}$, the depreciation was calculated over five years. Some studies included these costs in the total hospitalization cost, while others did not. In the study by Kaur et al. ${ }^{[1]}$, these costs were excluded since they were reported to be covered by philanthropic subsidies and assumed no extra cost to the public health system of Canada. In the population-based study by Swanson et al.$^{[9]}$, the cost that they reported incorporated the cost of the procedure to the hospital, but not the acquisition and annual maintenance cost of the robot.

In the prospective study by Gondé et al. ${ }^{[7]}$, total cost was defined by length of stay related costs (clinical expense, medical logistics, general logistics, and buildings) and costs independent of length of stay (direct charges including medical supplies and medico-technical expenses including capital depreciation). Part of the cost calculations in this study was based on the French National Cost Study database. In two population-based studies, Subramanian et al. ${ }^{[1]}$ and Paul et al. ${ }^{[10]}$ estimated costs by using total hospitalization charges and applying hospital-specific cost-to-charge ratios. It is unclear how cost was derived in the study by Glenn et al. ${ }^{[8]}$, another population-based study, which had the highest total cost $\left(102,057\right.$ USD) reported of all studies. In the study by Novellis et al. ${ }^{[14]}$, estimated cost was reported as percentage of regional health service reimbursement. This was derived from using actual costs as well as estimated costs.

\section{Cost comparison of robotic lobectomy to vats and open lobectomy}

Six of 16 studies compared the cost of robotic lobectomy to both VATS and open approaches [Table 2] $]^{[1,12,14-17]}$. Two studies found no significant difference in adjusted costs when comparing robotic approach to either VATS or open approach for the total hospital stay ${ }^{[16,17]}$; however, one of these studies noted that it may have been underpowered to detect a difference between groups ${ }^{[16]}$. Both studies used propensity score adjustment by inverse probability of treatment weighting. The study by Kneuertz et al ${ }^{[17]}$ did not find a difference in OR costs when comparing robotic to VATS (USD $9912 v s$. USD 9491; $P=0.44$ ); however, open approach had lower operating room costs than robotic (USD $8698 v s$. USD 9912; $P<0.01$ ). They observed an inverse relationship between OR related costs and postoperative related costs. Deen et al ${ }^{[15]}$ found that the overall cost for robotic approach was significantly higher than VATS $(\$ 17,011$ vs. $\$ 13,829 ; P<0.001)$, but did not find a significant difference when compared to open approach $(\$ 17,011 v s . \$ 15,036 ; P=0.058)$. 


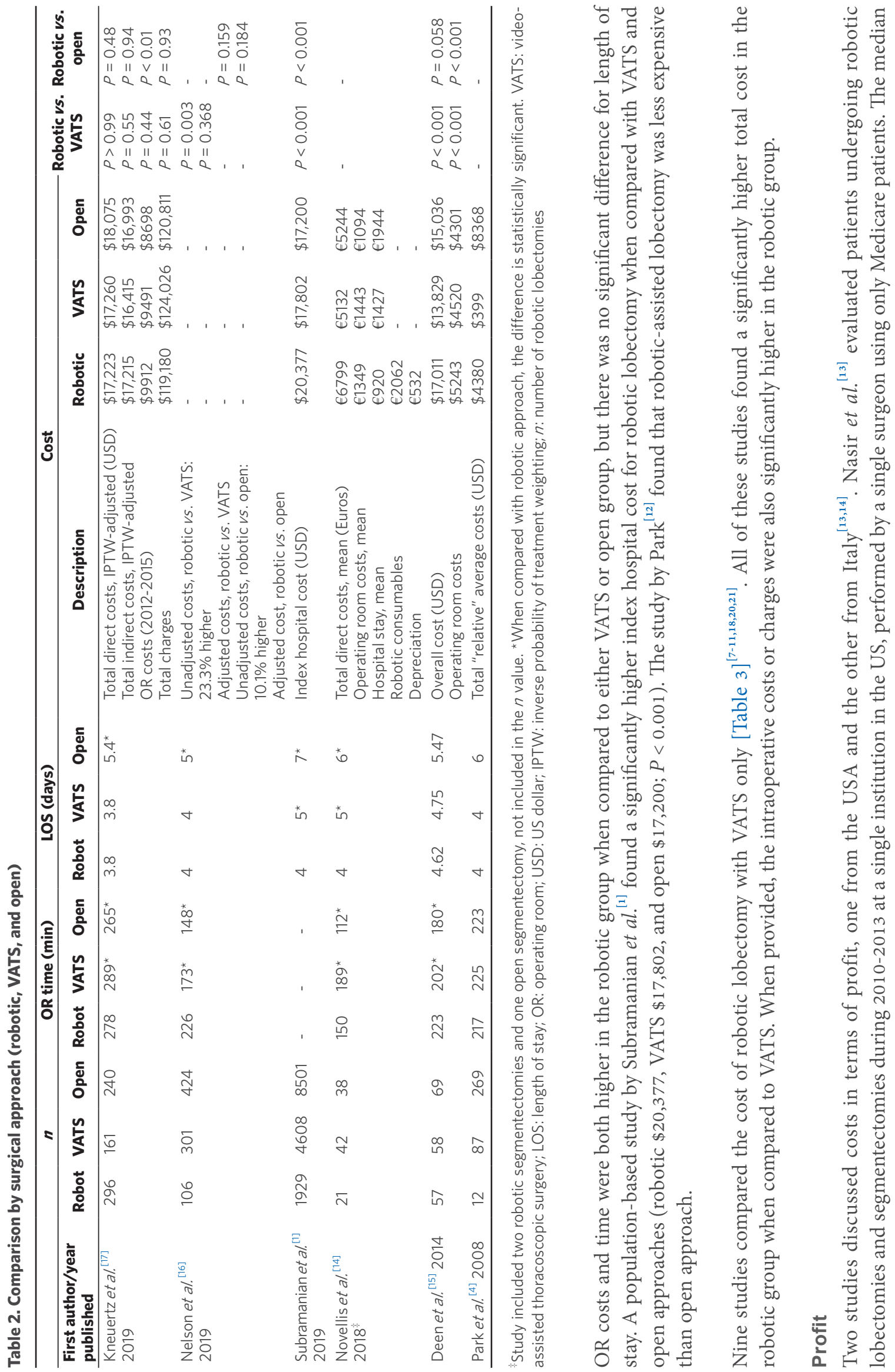




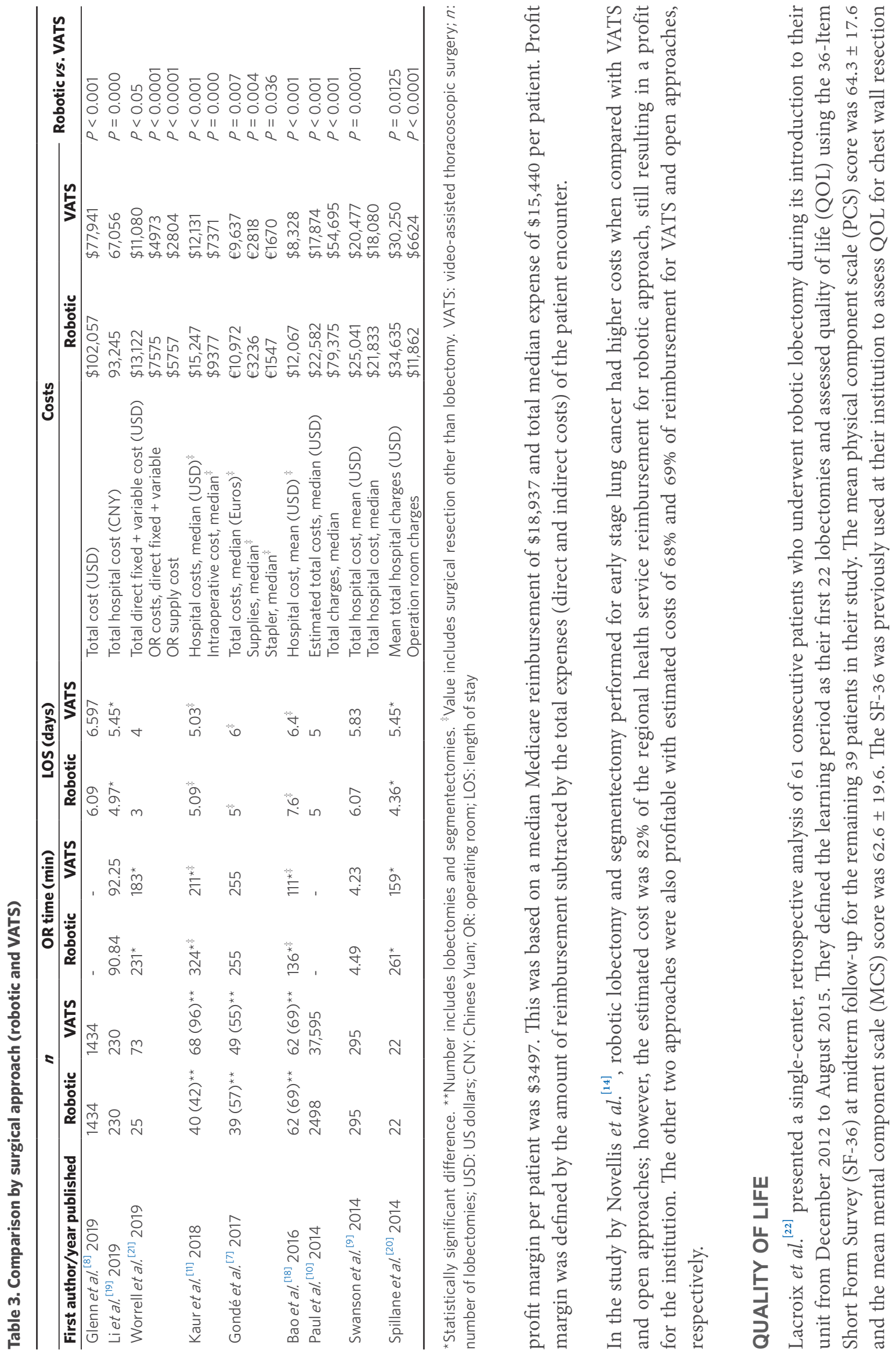


surgery, resulting in a mean PCS score of 40 and MCS score of 44. They found an association between pain and PCS scores, where PCS scores were significantly lower in patients with moderate pain (51.6 \pm 14.2) than those with mild $(69.4 \pm 17.7)$ or no pain $(67.8 \pm 16.1)(P=0.05)$. They concluded that QOL was satisfactory in their early experience for robotic lobectomy and was related to the pain level.

In the study by Worrell et al. ${ }^{[21]}$, costs and quality of life outcomes were evaluated during the initiation of their robotic lobectomy program. They compared their first 25 robotic assisted lobectomies with 73 VATS lobectomies, which were performed from 2010 to 2012. The European Organization for Research and Treatment of Cancer quality of life questionnaire (QLQ-30) was used to assess QOL with responses from 29 of the 98 patients, 9 robotic and 20 VATS, at a median follow-up of 65 months. This study found no significant difference between the robotic and VATS groups in their global health status and symptom scale median scores.

In a retrospective study, Cerfolio et al. ${ }^{[23]}$ reported a consecutive series of patients with clinically apparent resectable non-small cell lung cancer (NSCLC) from February 2010 to April 2011 who underwent attempted completely portal robot lobectomy using the four-arm technique. This group was compared against propensity-matched controls who underwent nerve- and rib-sparing thoracotomy. The study was performed by a single surgeon at a single institution. Quality of life information was obtained at two time points, three weeks and four months after surgery, and was measured by the Short Form Health Survey (SF-12) with supplemental questions about pain control. The robotic lobectomy group had a significantly higher average mental quality of life (MCS) score at three weeks when compared with the thoracotomy controls (53.5 vs. 40.3; $P<0.001$ ). A trend for higher physical quality of life (PCS) score at three weeks was observed with the robotic group, although it was not of statistical significance $(40.3 v s .43 .1 ; P=0.07)$. There was no significant difference observed for mental or physical quality of life at four months. The authors in this study noted that there may have been bias introduced in the surveys since the patients were informed that the robotic approach was a new and "less invasive" technique.

\section{DISCUSSION}

The hospital cost of robotic lobectomy during initiation of a robotic lobectomy program and/or early experiences at an institution has consistently been shown to be higher when compared to VATS lobectomy ${ }^{[11,18,20,21]}$. There were many factors observed to affect total hospital cost, one of which was intraoperative cost. Studies that disclosed OR time during early experiences reported a significantly longer time for robotic lobectomies when compared to VATS [Table 3] ${ }^{[1,18,20,21]}$. Two of these studies observed a decrease in operating time with more experience, which translated into a difference in intraoperative $\operatorname{cost}^{[1,20]}$. Kaur et al. ${ }^{[11]}$ found that, based on their micro-costing analysis, anatomic resections using the robotic approach cost more than VATS by $\$ 3116$ per case. They considered significantly higher intraoperative times to be a main contributor to this difference, and reported that OR time using the robotic platform decreased over time. There was a mean difference of $71 \mathrm{~min}(P=0.004)$ when comparing the first 20 robotic resections with the remaining 22 robotic resections, which resulted in an intraoperative cost difference of $\$ 883.38$, reducing the total hospital cost. In their study, Spillane et al ${ }^{[20]}$ attributed higher associated hospital charges for robotic-assisted lobectomies to increased cost of OR time. They also found a trend in a decrease in intraoperative duration with the robotic approach over time. In their study, Bao et al. ${ }^{[18]}$ noted that longer operative time for the robotic group may be due to the limited robotic experience of the surgeon.

This review also includes studies performed at centers with established robotic programs with high robotic surgical case volume. Case volume and surgeon experience may influence hospital costs. The amortized cost of robotic equipment is directly dependent on the number of cases performed, with higher volume 
resulting in lower costs. The two studies that unexpectedly demonstrated no significant difference in adjusted cost of robotic lobectomy compared to VATS were performed at high-volume surgical centers experienced in robotic surgery. These studies also found no significant difference in cost when comparing robotic to open lobectomy. Both Si and Xi systems were used and both reported on a more recent study period with patients evaluated into the year 2017.

There are also non-operating room costs to take into consideration. Postoperative complications have been shown to increase costs ${ }^{[16,24]}$. In Nelson's ${ }^{[16]}$ study, they reported an association between pulmonary and cardiovascular complications with increase in mean costs for all approaches. While the majority of studies in this review did not find a significant difference in overall postoperative major or minor complications between robotic and VATS or open groups ${ }^{[7,8,11,14-19,21]}$, this is a potential area for cost reduction. Kneuertz et al. ${ }^{[24]}$ performed a retrospective review of patients at our institution who underwent robotic-assisted lobectomy for NSCLC and evaluated postoperative outcomes on cost. Postoperative complications and prolonged hospital stay added considerable hospital expenses, which was the largest variability in total cost in the study.

The studies in this review that reported a difference in postoperative complications between groups were multi-institutional database studies ${ }^{[1,9]}$. Swanson et al. ${ }^{[9]}$ reported that patients undergoing lobectomy via robotic approach from 2009 to 2011 were 4.24 times more likely to have a minor event than those undergoing VATS. In contrast, the study by Subramanian et al. ${ }^{[1]}$ found that, from 2009 to 2014, robotic lobectomy compared with VATS was associated with decreased adjusted risk of any minor postoperative complication, and, when compared with the open approach, had a decreased risk of any major or minor postoperative complication. Glenn et al. ${ }^{[8]}$ found no significant difference in overall morbidity between the robotic group and VATS group from 2010 to 2013; however, they observed that, in the earlier period of the study (2010-2011), morbidity was significantly higher in the robotic group when compared with VATS (robotic $42.9 \% v s$. VATS $36.3 \%, P=0.004$ ). From 2012 to 2013, there was no longer a significant difference. Findings in these studies suggest, but do not confirm, that postoperative complications may be higher in earlier experiences of robotic lobectomy.

Based on the literature comparing all three approaches at single institutions, the cost of robotic lobectomy appears to be comparable if not less costly than open lobectomy and/or profitable. While OR time was significantly longer in the robotic group in these studies, length of stay was shorter or similar. The reduction in length of stay was noted by some authors to account for their findings. From the three studies that evaluated quality of life in their early experience, it appears that the robotic approach has acceptable results, although the number of studies and patients evaluated are limited ${ }^{[21-23]}$.

Many studies in our review compared robotic approach to VATS only, with results consistently demonstrating higher costs for robotic lobectomy. Interestingly, no study was identified during our literature search that compared costs for robotic approach to thoracotomy only even when the data suggest that the continued decline in thoracotomy for lobectomies appears mainly a result from increased adoption of the robotic platform not from increased use of VATS ${ }^{[1,2]}$. While the majority of studies show that robotic lobectomy has higher hospital costs than VATS, the significance of this finding is unclear. The difference in index hospital cost is of statistical significance, but its overall impact on patient outcomes and health economics has not been elucidated and the value of using the robotic platform has not been defined. Further studies on patient outcomes such as quality of life, recovery time, and morbidity, as well as surgeon factors, are needed.

\section{Study limitations}

There are limitations of this study. Due to the heterogeneity of how costs were defined and analyzed, a quantitative analysis is not feasible in this study and direct comparisons between studies could not be 
performed. There appears to be an overall underappreciation in the surgical literature of the differences among cost, charges, and recovery of services, which rendered comparison incredibly difficult. Additionally, there is little appreciation for the running costs that go into caring for these patients and are often assumed into operational overhead. This review was also based on observational studies, with all but one study utilizing retrospective analysis. In addition, the majority of studies reported using the $\mathrm{Si}$, which is an older generation. Only two of 16 studies reviewed noted using the Xi, which was Food and Drug Administration approved and introduced to the USA in 2014. Another limitation is the limited number of studies regarding quality of life available for review. More studies on patient reported outcomes for those undergoing robotic lobectomies are needed to better understand its impact on quality of life. Finally, while we evaluated financial costs to the hospital and quality of life of patients undergoing robotic lobectomy, we did not comprehensively assess the value of the robotic platform. There are more important factors to consider beyond index hospitalization costs and PRO.

\section{CONCLUSION}

Developing a robotic lobectomy program may be associated with relatively higher index hospital costs when compared to VATS approach. With increased experience and volume of robotic cases, this difference may no longer be of significance, but additional defining of costs versus charges is needed as a surgical society. As an overall review, the cost of robotic lobectomy is comparable if not less costly than open lobectomy based on single institution studies and may be profitable for the hospital, if we can better understand the operational costs needed to care for these patients. Quality of life appears to be acceptable in the early experience of robotic lobectomy.

\section{DECLARATIONS}

Authors' contributions

Manuscript preparation: Nishimura JM

Editorial of manuscript: Nishimura JM, Goodwin M, Kneuertz P, Moffatt-Bruce S, Merritt RE, D’Souza DM

\section{Availability of data and materials}

Not applicable.

\section{Financial support and sponsorship}

None.

\section{Conflicts of interest}

Dr. Desmond D'Souza is a proctor for Intuitive Surgical Inc. Dr. Robert Merritt is a speaker for Intuitive Surgical Inc. All other authors declare no conflicts of interest.

\section{Ethical approval and consent to participate}

Not applicable.

\section{Consent for publication}

Not applicable.

\section{Copyright}

(c) The Author(s) 2020.

\section{REFERENCES}

1. Subramanian MP, Liu J, Chapman Jr WC, Olsen MA, Yan Y, et al. Utilization trends, outcomes, and cost in minimally invasive lobectomy. Ann Thorac Surg 2019;108:1648-55. 
2. Oh DS, Reddy RM, Gorrepati ML, Mehendale S, Reed MF. Robotic-assisted, video-assisted thoracoscopic and open lobectomy: propensity-matched analysis of recent premier data. Ann Thorac Surg 2017;104:1733-40.

3. Veronesi G, Galetta D, Maisonneuve P, Melfi F, Schmid RA, et al. Four-arm robotic lobectomy for the treatment of early-stage lung cancer. J Thorac Cardiovasc Surg 2010;140:19-25.

4. Park BJ, Flores RM, Rusch VW. Robotic assistance for video-assisted thoracic surgical lobectomy: technique and initial results. J Thorac Cardiovasc Surg 2006;131:54-9.

5. Dylewski MR, Ohaeto AC, Pereira JF. Pulmonary resection using a total endoscopic robotic video-assisted approach. Semin Thorac Cardiovasc Surg 2011;23:36-42.

6. Singer E, Kneuertz PJ, D’Souza DM, Moffatt-Bruce SD, Merritt RE. Understanding the financial cost of robotic lobectomy: calculating the value of innovation? Ann Cardiothorac Surg 2019;8:194-201.

7. Gondé H, Laurent M, Gillibert A, Sarsam OM, Varin R, et al. The affordability of minimally invasive procedures in major lung resection: a prospective study. Interact Cardiovasc Thorac Surg 2017;25:469-75.

8. Glenn ZF, Zubair M, Hussain L, Grannan K. Comparison of pulmonary lobectomies using robotic and video-assisted thoracoscopic approaches: results from 2010-2013 national inpatient sample. J Cardiovasc Surg (Torino) 2019;60:526-31.

9. Swanson SJ, Meyers BF, Gunnarsson CL, Moore M, Howington JA, et al. Video-assisted thoracoscopic lobectomy is less costly and morbid than open lobectomy: a retrospective multiinstitutional database analysis. Ann Thorac Surg 2012;93:1027-32.

10. Paul S, Jalbert J, Isaacs AJ, Altorki NK, Isom OW, et al. Comparative effectiveness of robotic-assisted vs. thoracoscopic lobectomy. Chest 2014;146:1505-12.

11. Kaur MN, Xie F, Shiwcharan A, Patterson L, Shargall Y, et al. Robotic versus video-assisted thoracoscopic lung resection during early program development. Ann Thorac Surg 2018;105:1050-7.

12. Park BJ, Flores RM. Cost comparison of robotic, video-assisted thoracic surgery and thoracotomy approaches to pulmonary lobectomy. Thorac Surg Clin 2008;18:297-300

13. Nasir BS, Bryant AS, Minnich DJ, Wei B, Cerfolio RJ. Performing robotic lobectomy and segmentectomy: cost, profitability, and outcomes. Ann Thorac Surg 2014;98:203-8.

14. Novellis P, Bottoni E, Voulaz E, Cariboni U, Testori A, et al. Robotic surgery, video-assisted thoracic surgery, and open surgery for early stage lung cancer: comparison of costs and outcomes at a single institute. J Thorac Dis 2018;10:790-8.

15. Deen SA, Wilson JL, Wilshire CL, Vallières E, Farivar AS, et al. Defining the cost of care for lobectomy and segmentectomy: a comparison of open, video-assisted thoracoscopic, and robotic approaches. Ann Thorac Surg 2014;97:1000-7.

16. Nelson DB, Mehran RJ, Mitchell KG, Rajaram R, Correa AM, et al. Robotic-assisted lobectomy for non-small cell lung cancer: a comprehensive institutional experience. Ann Thorac Surg 2019;108:370-6.

17. Kneuertz PJ, Singer E, D'Souza DM, Abdel-Rasoul M, Moffatt-Bruce SD, et al. Hospital cost and clinical effectiveness of roboticassisted versus video-assisted thoracoscopic and open lobectomy: a propensity score-weighted comparison. J Thorac Cardiovasc Surg 2019;157:2018-26.e2.

18. Bao F, Zhang C, Yang Y, He Z, Wang L, et al. Comparison of robotic and video-assisted thoracic surgery for lung cancer: a propensitymatched analysis. J Thorac Dis 2016;8:1798-803.

19. Li JT, Liu PY, Huang J, Lu PJ, Lin H, et al. Perioperative outcomes of radical lobectomies using robotic-assisted thoracoscopic technique vs. video-assisted thoracoscopic technique: retrospective study of 1,075 consecutive p-stage I non-small cell lung cancer cases. J Thorac Dis 2019;11:882-91.

20. Spillane J, Brooks P. Developing a robotic program in thoracic surgery at Cape Cod Hospital. J Robot Surg 2014;8:213-20.

21. Worrell SG, Dedhia P, Gilbert C, James C, Chang AC, et al. The cost and quality of life outcomes in developing a robotic lobectomy program. J Robot Surg 2019;13:239-43.

22. Lacroix V, Mosala Nezhad Z, Kahn D, Steyaert A, Poncelet A, et al. Pain, quality of life, and clinical outcomes after robotic lobectomy. Thorac Cardiovasc Surg 2017;65:344-50.

23. Cerfolio RJ, Bryant AS, Skylizard L, Minnich DJ. Initial consecutive experience of completely portal robotic pulmonary resection with 4 arms. J Thorac Cardiovasc Surg 2011;142:740-6.

24. Kneuertz PJ, Singer E, D'Souza DM, Moffatt-Bruce SD, Merritt RE. Postoperative complications decrease the cost-effectiveness of robotic-assisted lobectomy. Surgery 2019;165:455-60. 